\title{
Partial $J_{2}$-Invariance for Spacecraft Formations
}

\author{
Louis Breger* and Jonathan P. How ${ }^{\dagger}$ \\ MIT Department of Aeronautics and Astronautics \\ Kyle T. Alfriend $\ddagger$ \\ Texas A\&M University Department of Aerospace Engineering
}

\begin{abstract}
This paper presents a method for finding spacecraft formation initial conditions (ICs) that minimize the drift resulting from $J_{2}$ disturbances and also minimize the fuel required to attain those ICs. A third goal that the formation remain in a particular geometry can also be considered. The approach uses linear optimization, but is valid for highly eccentric and widely spaced orbits. Optimization allows for the intelligent selection of degrees of freedom in already existing invariance conditions, as well as the minimization of different types of drift. Results are compared to $J_{2}$-invariance conditions in the literature and the method is shown to find relative orbits with slightly lower levels of drift that require significantly less $\Delta V$ to obtain.
\end{abstract}

\section{Introduction}

One of the principle requirements of a spacecraft formation is that the component spacecraft do not drift apart from one another. ${ }^{6}$ In a fully Keplerian orbit, the only source of drift over multiple orbits is a difference between spacecraft periods, which is equivalent to a difference in spacecraft semimajor axes. ${ }^{7}$ The presence of the relative disturbances between spacecraft (e.g., relative drag, $J_{2}$ ) can also lead to drift in a formation. An alternative to expending regular control energy to counteract drift is to choose formation initial conditions that reduce relative drift between spacecraft. This paper presents a method for finding spacecraft formation initial conditions (ICs) that minimize the drift resulting from $J_{2}$ disturbances and also minimize the fuel required to attain those ICs while approximating a specified formation configuration.

Several approaches for creating $J_{2}$ invariant relative orbits have recently been proposed in the literature. $^{4,2,8}$ Different classes of "invariant" orbits have been introduced: those that are truly invariant over time, orbits that retain the same mean period over time, orbits that are invariant except for argument of perigee drift, and orbits that are invariant except for right ascension drift. In the case of full invariance conditions, where the formation returns to an identical relative state every orbit, the set of relative orbits that satisfy the conditions is very small and the geometry

\footnotetext{
${ }^{*}$ Research Assistant, MIT Department of Aeronautics and Astronautics, lbreger@mit.edu

${ }^{\dagger}$ Associate Professor, MIT Department of Aeronautics and Astronautics, jhow@mit.edu

${ }^{\ddagger}$ Professor, Texas A\&M University Department of Aerospace Engineering, alfriend@aeromail.tamu.edu
} 
of those orbits is highly restricted. ${ }^{4}$ Hence, it is more common for a $J_{2}$-invariant orbit to only be invariant in a reduced set of dimensions for which it is possible to analytically cancel the relative effects of $J_{2}$. In all of the invariance cases, the drift being minimized is secular variation in the mean orbital elements.

The approach presented in this paper uses convex linear optimization techniques to find initial conditions that minimize drift due to relative $J_{2}$ effects. The optimization balances the objective of reducing drift (in a Cartesian sense), against the objectives to minimize the fuel use required to achieve the initial conditions and maintain a specific formation geometry. This approach can be used both to initialize a newly deployed formation that is not yet in a $J_{2}$-invariant orbit and to re-initialize a formation that has drifted from its original orbit. Optimization allows for the selection of degrees of freedom in already existing invariance conditions in a way that is cognizant of other problem objectives. In addition, optimizing allows for partial combinations of types of $J_{2^{-}}$ invariance that enable alternate types of drift to be minimized or even to allow some drift in favor of improved fuel use. This is an attractive prospect when one considers that it is unlikely a precise initial condition will ever be achieved (i.e., errors will result from sensing, imperfect thrusting) and the orbits will need to be re-initialized regularly.

The formulation for minimizing maneuver fuel expenditures in Ref. 1 is used in combination with the $J_{2}$-modified state transition matrices presented in Ref. 3 . The optimizations are solved very rapidly and can be used online to optimize conditions for Earth-orbiting formation flying missions (e.g., LEO, HEO) that require drift be minimized, but that also have particular geometry requirements such as separation distance or shape (e.g., $\left.\mathrm{MMS}^{10}\right)$. Results in this paper show that levels of drift similar to those achievable through invariance conditions in the literature are obtainable, while simultaneously showing the minimum amount of fuel required to obtain them. The effects of weighting geometry highly are also shown.

\section{Optimizing Invariance}

The osculating orbital element difference $\delta \mathbf{e}$ between two orbits is

$$
\delta \mathbf{e}(t)=\mathbf{e}_{B}(t)-\mathbf{e}_{A}(t)
$$

where $\mathbf{e}_{A}(t)$ and $\mathbf{e}_{B}(t)$ are absolute osculating orbital elements. The two orbits are invariant if $\delta \mathbf{e}$ remains unchanged over a period of time, so that $\delta \mathbf{e}\left(t_{1}\right) \equiv \delta \mathbf{e}\left(t_{2}\right)$, where $t_{2}-t_{1}$ is the duration of interest (typically an integer number of orbits). The relative state between the two spacecraft can be propagated including the effects of $J_{2}$ using the state propagation matrix in Ref. 3,

$$
\delta \mathbf{e}\left(t_{2}\right)=D^{-1}\left(\mathbf{e}_{A}\left(t_{2}\right)\right) \bar{\Phi}^{*}\left(\mathbf{e}_{A}\left(t_{1}\right), t_{2}, t_{1}\right) D\left(\mathbf{e}_{A}\left(t_{1}\right)\right) \delta \mathbf{e}\left(t_{1}\right)
$$

where $t_{1}$ and $t_{2}$ are times, $\bar{\Phi}^{*}$ is a state transition matrix for mean orbital element differences, $D$ is a linearized matrix that rotates from osculating orbital element differences to mean orbital element differences, and $D^{-1}$ is a linearized matrix that rotates from mean orbital element differences to 
osculating orbital element differences. Using the state transition matrix from Eq. (2) gives the constraint

$$
\delta \mathbf{e}\left(t_{1}\right)=\delta \mathbf{e}\left(t_{2}\right)=D^{-1}\left(\mathbf{e}\left(t_{2}\right)\right) \bar{\Phi}^{*}\left(\mathbf{e}\left(t_{1}\right), t_{2}, t_{1}\right) D\left(\mathbf{e}\left(t_{1}\right)\right) \delta \mathbf{e}\left(t_{1}\right)
$$

Defining the matrix function $\bar{\Phi}_{D k}^{*} \equiv D^{-1}\left(\mathbf{e}\left(t_{k+1}\right)\right) \bar{\Phi}^{*}\left(\mathbf{e}\left(t_{k}\right), t_{k+1}, t_{k}\right) D\left(\mathbf{e}\left(t_{k}\right)\right)$ gives the invariance condition

$$
\delta \mathbf{e}\left(t_{1}\right)=\bar{\Phi}_{D 1}^{*} \delta \mathbf{e}\left(t_{1}\right) \quad \rightarrow \quad\left(\bar{\Phi}_{D 1}^{*}-I\right) \delta \mathbf{e}\left(t_{1}\right)=0
$$

where $I$ is a $6 \times 6$ identity matrix. As mentioned above, the resulting geometry of the no-drift (complete invariance) condition is too restrictive for many missions, but partially invariant conditions can be obtained by minimizing the weighted norm of the invariance condition

$$
\min _{\delta \mathbf{e}\left(t_{1}\right)}\left\|W_{d}\left(\bar{\Phi}_{D 1}^{*}-I\right) \delta \mathbf{e}\left(t_{1}\right)\right\|
$$

where the weighting matrix $W_{d}$ is introduced to extract states of interest to penalize particular types of drift. To penalize position and velocity states, use the matrix $M(\mathbf{e}(t))$ where

$$
M \mathbf{x}=\delta e_{\mathrm{osc}}
$$

and the analytic form of $M$ can be found in Ref. 9. The vector $\mathbf{x}$ is in the LVLH coordinate system and has the form

$$
\mathbf{x}=\left[\begin{array}{llllll}
x & y & z & \dot{x} & \dot{y} & \dot{z}
\end{array}\right]^{T}
$$

where the positions $x, y$, and $z$ are in meters and the velocities $\dot{x}, \dot{y}$, and $\dot{z}$ are in meters per second. If $W_{d}$ is chosen to be the matrix $M(\mathbf{e}(t))$, then the elements of the LVLH state can be directly penalized (e.g., extracting only position states could penalize meters of drift). This enables the drift formulation to penalize the distance from the desired geometry in a Cartesian frame, as opposed to just using orbital elements. Penalizing true separation distance finds initial conditions that will maintain the formation shape, an important consideration for missions that require specific geometric configurations. ${ }^{10}$

The overall problem statement then is, given a spacecraft at offset $\delta \mathbf{e}\left(t_{0}\right)$, design a control input sequence $U(\tau), \tau \in\left[t_{0}, t_{1}\right]$ that generates a set of initial conditions at $t_{1}$ that balances the trade-off between the ensuing drift by time $t_{2}$, the fuel cost of achieving these initial conditions, and the extent to which the formation geometry is maintained. In order to formulate the full problem statement, a method for incorporating inputs must be introduced. Inputs to the system for any given time step $k$ are

$$
\mathbf{u}_{k}=\left[\begin{array}{lll}
u_{x_{k}} & u_{y_{k}} & u_{z_{k}}
\end{array}\right]^{T}
$$

where $u_{x_{k}}, u_{y_{k}}$, and $u_{z_{k}}$ are the inputs in the axes indicated by the subscripts in the LVLH frame. 
The effects of an input $\mathbf{u}_{k}$ from step $k$ to step $k+1$ is given the discrete input effect matrix $\Gamma$

$$
\Gamma=\int_{t_{k}}^{t_{k+1}}\left\{D^{-1}\left(\mathbf{e}_{d}\left(t_{k+1}\right)\right) \bar{\Phi}^{*}\left(\mathbf{e}_{m d}(\tau), t_{k+1}, \tau\right) D\left(\mathbf{e}_{d}(\tau)\right) M\left(\mathbf{e}_{d}(\tau)\right)\left[\begin{array}{c}
0_{3} \\
I_{3}
\end{array}\right]\right\} d \tau
$$

where $t_{k}$ is the time corresponding to step $k$. A number of approximate methods for computing $\Gamma$ are presented and evaluated in Ref. 5 . The inputs over a plan of duration $t_{1}-t_{0}$ with $N$ discrete steps are given by the plan input vector $U$

$$
U=\left[\begin{array}{lllll}
\mathbf{u}_{0}^{T} & \mathbf{u}_{1}^{T} & \ldots & \mathbf{u}_{N-2}^{T} & \mathbf{u}_{N-1}^{T}
\end{array}\right]^{T}
$$

The effect of all the inputs in $U$ is found using a discrete convolution matrix

$$
\hat{\Gamma}=\left[\begin{array}{lllll}
\tilde{\Phi}(n, 1) \tilde{\Gamma}(0) & \tilde{\Phi}(n, 2) \tilde{\Gamma}(1) \quad \ldots \quad \tilde{\Phi}(n, n-1) \tilde{\Gamma}(n-2) \quad \tilde{\Gamma}(n-1)
\end{array}\right]
$$

where $\tilde{\Phi}(k, j) \equiv D^{-1}\left(\mathbf{e}\left(k t_{s}\right)\right) \bar{\Phi}^{*}\left(\mathbf{e}, k t_{s}, j t_{s}\right) D\left(\mathbf{e}\left(j t_{s}\right)\right), \tilde{\Gamma}(k) \equiv \Gamma\left(\mathbf{e},(k+1) t_{s}, k t_{s}\right)$, and $t_{s}$ is the discretization time step. The semi-invariant initial condition optimization cost function is

$$
C^{*}=\min _{U} Q_{d}\left\|W_{d}\left(\bar{\Phi}_{D 1}^{*}-I\right)\left(\bar{\Phi}_{D 0}^{*} \delta \mathbf{e}\left(t_{0}\right)+\hat{\Gamma} U\right)\right\|+Q_{x}\left\|W_{x} \hat{\Gamma} U\right\|+Q_{u}\|U\|
$$

where $C^{*}$ is the optimal cost, $W_{x}$ is a weighting matrix to specify the type of geometry penalty, $Q_{u}$ is a weighting on fuel minimization, $Q_{x}$ is a weighting on desired formation geometry, and $Q_{d}$ is a weighting on drift. The cost function uses the initial state of each spacecraft in the formation as the desired geometry, so the geometry weighting penalizes deviations from the openloop state propagation. Note that a simple modification to the cost function could separate the initial geometry from the desired geometry. The optimization in Eq. (12) can be easily implemented as a linear program if 1-norms are used, permitting efficient, fast online solutions. ${ }^{11}$ As expected, a sufficiently high weighting on invariance results in a minimizing control input $U^{*}$ where $\hat{\Gamma} U^{*}=$ $-\bar{\Phi}_{D 0}^{*} \delta \mathbf{e}\left(t_{0}\right)$. Alternately, a sufficiently high $Q_{x}$ (with an identity matrix for $W_{x}$ ) results in $\hat{\Gamma} U^{*}=$ $\left[\begin{array}{llllll}0 & 0 & 0 & 0 & 0 & 0\end{array}\right]^{T}$, because the inputs will all be zero in order to maintain the original geometry.

The cost function in Eq. (12) could be optimized using any one of a variety of methods. To formulate the optimization as a linear program, 1-norms is used, yielding

$$
C^{*}=\min _{U} Q_{d}\left\|W_{d}\left(\bar{\Phi}_{D 1}^{*}-I\right)\left(\bar{\Phi}_{D 0}^{*} \delta \mathbf{e}\left(t_{0}\right)+\hat{\Gamma} U\right)\right\|_{1}+Q_{x}\|\hat{\Gamma} U\|_{1}+Q_{u}\|U\|_{1}
$$

\section{Results}

The cost function in Eq. (12) was used to find optimized $J_{2}$ invariant conditions for several examples. To compare the method in this paper to invariance conditions in the literature, the LEO orbit from the example in Ref. 2 is used

$$
\mathbf{e}_{A}\left(t_{0}\right)=\left[\begin{array}{llllll}
1.11448077 & 0.005547 & 1.22194 & 0 & 0 & 0
\end{array}\right]^{T}
$$




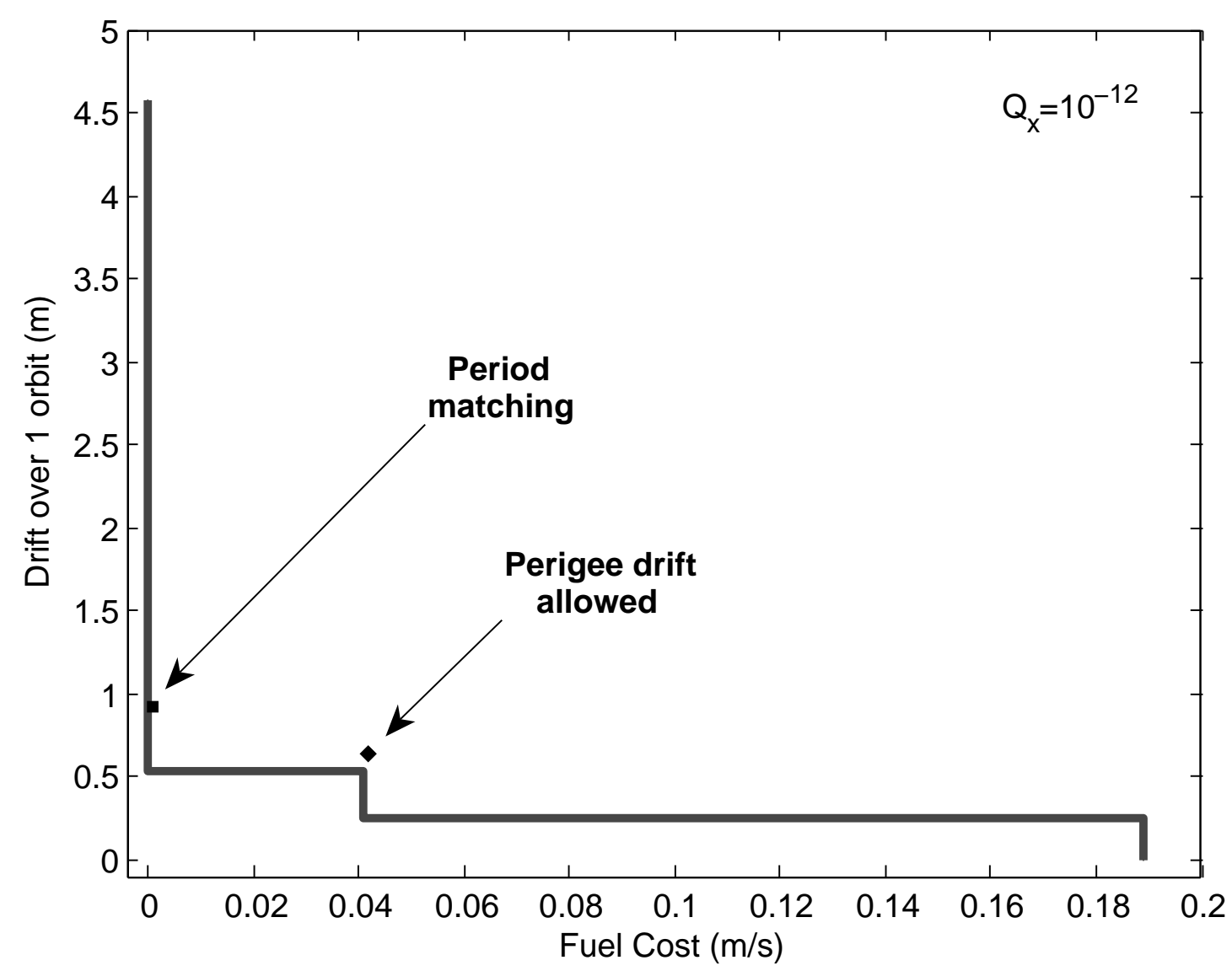

Fig. 1: Expected drift due to differential $J_{2}$ effects and fuel cost of a range of optimized initial conditions in a LEO orbit. The lines between marked points indicate the least drift that can be attained for the indicated amount of fuel. In this example the fuel weighting is 1 , the geometry weighting is $10^{-12}$, and the drift weighting is allowed to vary widely. The red square represents the solution based on the period-matching condition in Case 1 of Ref. 2. The black diamond represents the solution based on the $J_{2}$ invariant orbit with perigee drift in Ref. 4 .

where the elements of $\mathbf{e}_{A}$ are semimajor axis (normalized by Earth's radius), eccentricity, inclination (radians), right ascension of the ascending node (radians), argument of perigee, and mean anomaly, respectively. The osculating relative state of the $\mathbf{e}_{B}$ with respect to $\mathbf{e}_{A}$ at the initial time, $t_{0}$, is

$$
\zeta\left(t_{0}\right)=\left[\begin{array}{r}
6.00000 \times 10^{-8} \\
2.03604 \times 10^{-6} \\
5.50000 \times 10^{-6} \\
5.19093 \times 10^{-6} \\
-3.60421 \times 10^{-3} \\
3.55449 \times 10^{-3}
\end{array}\right]
$$

Figure 1 shows drift rates due to differential $J_{2}$ effects and fuel costs for a series of initial conditions generated by the optimization method with a half orbit planning horizon as $Q_{d}$ is changed. For this example, the 1-norm is used to penalize both drift and fuel use and both $W_{d}$ 


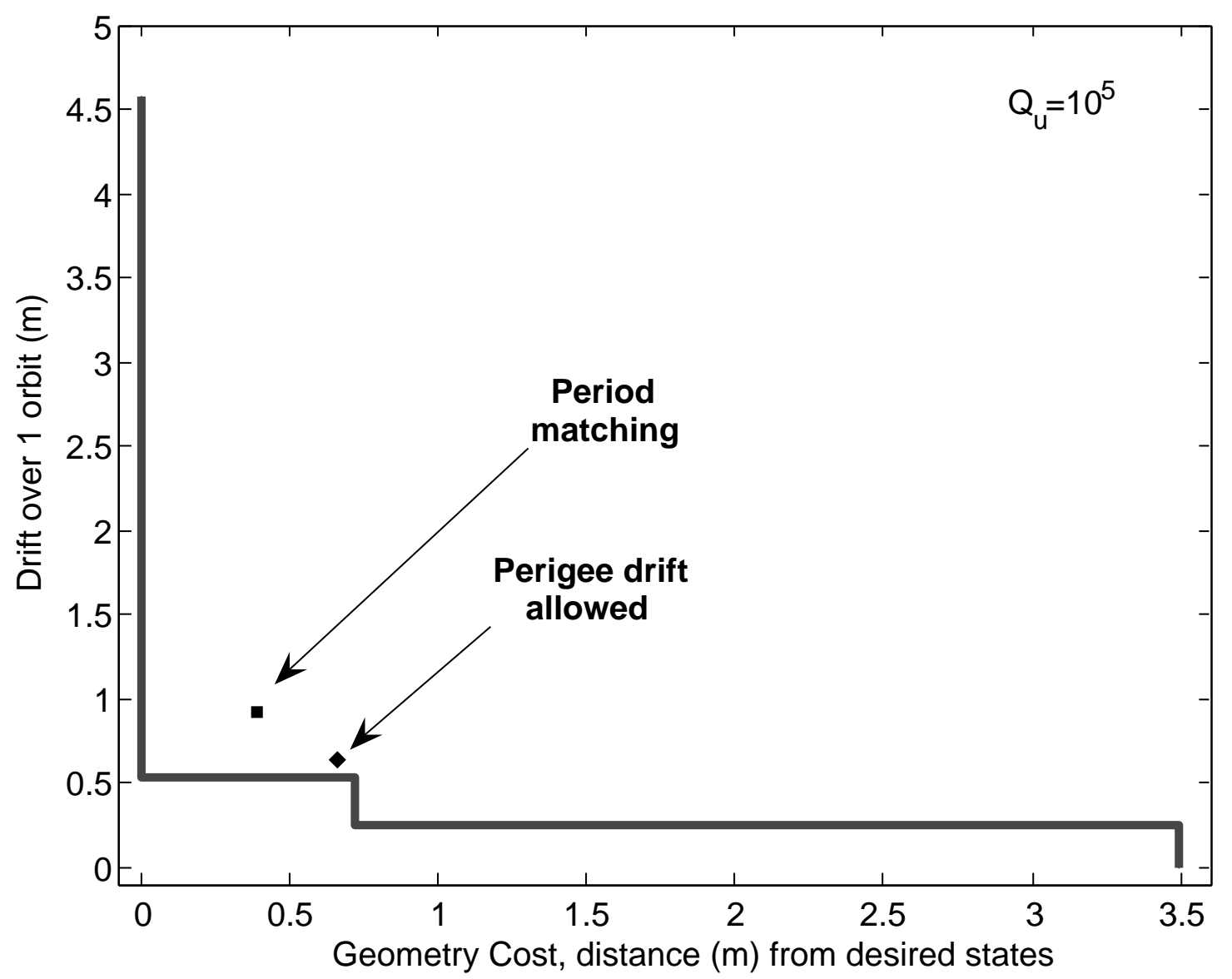

Fig. 2: Expected drift due to differential $J_{2}$ effects and geometry cost of a range of optimized initial conditions in a LEO orbit. The lines between marked points indicate the least drift that can be attained for the indicated amount of separation from the desired orbital offset.

and $W_{x}$ are set to the position rows of $M$ in order to only penalize position drift and geometry separation. With a very low $Q_{d}, Q_{u}$ will dominate, resulting in no control use. The zero drift point corresponds to high fuel use, because it necessitates driving the spacecraft to nearly the same orbits. A range of possible optimized initial conditions lie in between those extrema. The high-drift, unmodified initial conditions lie very close to the vertical drift axis, but drop to just over $0.5 \mathrm{~m}$ of drift with a minimum of fuel use. Further drift reductions are possible, but at greater fuel cost. It is readily apparent from the graph that using additional $\Delta V$ will produce diminishing returns in terms of reducing drift.

Initial conditions generated by other $J_{2}$-invariant conditions should lie either on or above the optimized result. The $\boldsymbol{\square}$ in Fig. 1 represents the initial condition based on the $J_{2}$ invariance condition in Case 1 of Ref. 2 that requires no mean period drift. The point is nearly optimal for this example, but this is not guaranteed to be the case for other problems. The indicates the drift that occurs when using semi-invariant initial conditions that allow perigee drift. ${ }^{4}$ In both specific cases, the partial invariance conditions allow for a range of possible initial conditions. Both analytic cases occur near the same drift levels as the initial conditions found by the optimizing approach. This indicates the optimized ICs may, in those cases, be meeting the same invariance 
criteria, while simultaneously finding ICs that minimize fuel use. The optimization-based approach enables the identification of a range of fuel-optimized initial conditions that can be used to better meet the requirements of a specific mission.

Figure 2 shows a number of optimizations of the same orbit and desired offset, however, in this case, both $Q_{x}$ and $Q_{u}$ are made significant while $Q_{d}$ is varied. The figure shows the cost associated with changing the formation geometry $\left(\left\|W_{x} \hat{\Gamma} U\right\|_{1}\right)$ versus the fuel cost $\left(\|U\|_{1}\right)$. When $Q_{x}$ is very high relative to $Q_{d}$, the optimized ICs, $\delta \mathbf{e}\left(t_{1}\right)$, are equivalent to the open-loop propagation of $\delta \mathbf{e}\left(t_{0}\right)$ (i.e., no fuel is used). This corresponds to $4.5 \mathrm{~m}$ of drift over an orbit. As $Q_{d}$ is increased, the optimized ICs are farther from $\bar{\Phi}_{D 0}^{*} \delta \mathbf{e}\left(t_{0}\right)$, but the resulting drift is lower because $Q_{d}$ has a greater effect on the solution. The solution that achieves $0.5 \mathrm{~m}$ of drift with almost no geometry cost represents a compromise between the desired formation geometry and the drift resulting from the effects of relative $J_{2}$. At the cost of slightly repositioning the formation (velocity changes are not penalized in the $W_{d}$ used for this example), the drift over an orbit has been reduced by $4 \mathrm{~m}$. When invariance dominates (the lower-right corner of the figure), the optimized initial conditions cancel almost all of the orbital offset $\delta \mathbf{e}$, indicating that geometry goals have been ignored.

Figures 3 and 4 show the effect of independently varying the fuel and geometry weights for an MMS-like HEO orbit

$$
\mathbf{e}_{A}\left(t_{0}\right)=\left[\begin{array}{llllll}
6.6 & 0.82 & 0.17 & 6.28 & 6.28 & 3.14
\end{array}\right]^{T}
$$

with the desired geometry

$$
\zeta\left(t_{0}\right)=\left[\begin{array}{r}
-4.00000 \times 10^{-8} \\
-4.98086 \times 10^{-7} \\
-8.50000 \times 10^{-7} \\
2.30000 \times 10^{-7} \\
-7.33646 \times 10^{-7} \\
3.72778 \times 10^{-6}
\end{array}\right]
$$

As in the LEO case, the unmodified initial conditions experience a significant amount of drift, which can be greatly reduced using comparatively small fuel expenditures. Likewise, small (centimeter) changes in the formation geometry can also decrease the drift. In this case, more intermediate optimized geometry steps exist, allowing for additional choices between precisely achieving the desired shape and drifting out of that shape over the course of the next orbit.

\section{Conclusions}

This paper introduced an approach to optimizing $J_{2}$ invariance between spacecraft that explicitly minimized the fuel use required to achieve the invariant states. This approach also allowed weights to be assigned the emphasis on invariance (i.e., preventing drift), minimizing fuel use, and maintaining a desired geometry. A Low Earth Orbit example showed that this optimized approach can produce results very similar to those that can be obtained by applying analytic invariance con- 


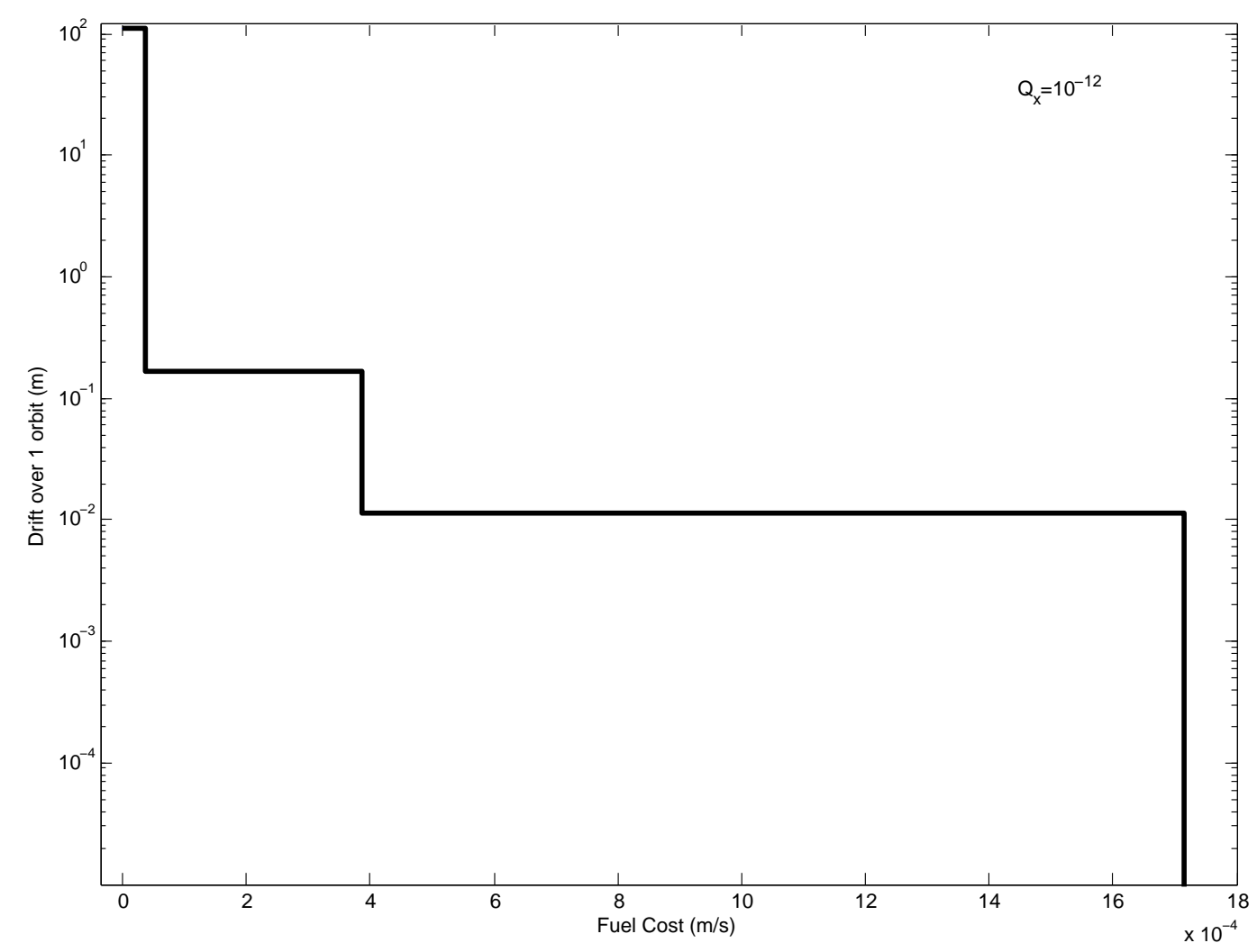

Fig. 3: Expected drift due to differential $J_{2}$ effects and fuel cost of a range of optimized initial conditions in a HEO orbit. The lines between marked points indicate the least drift that can be attained for the indicated amount of fuel.

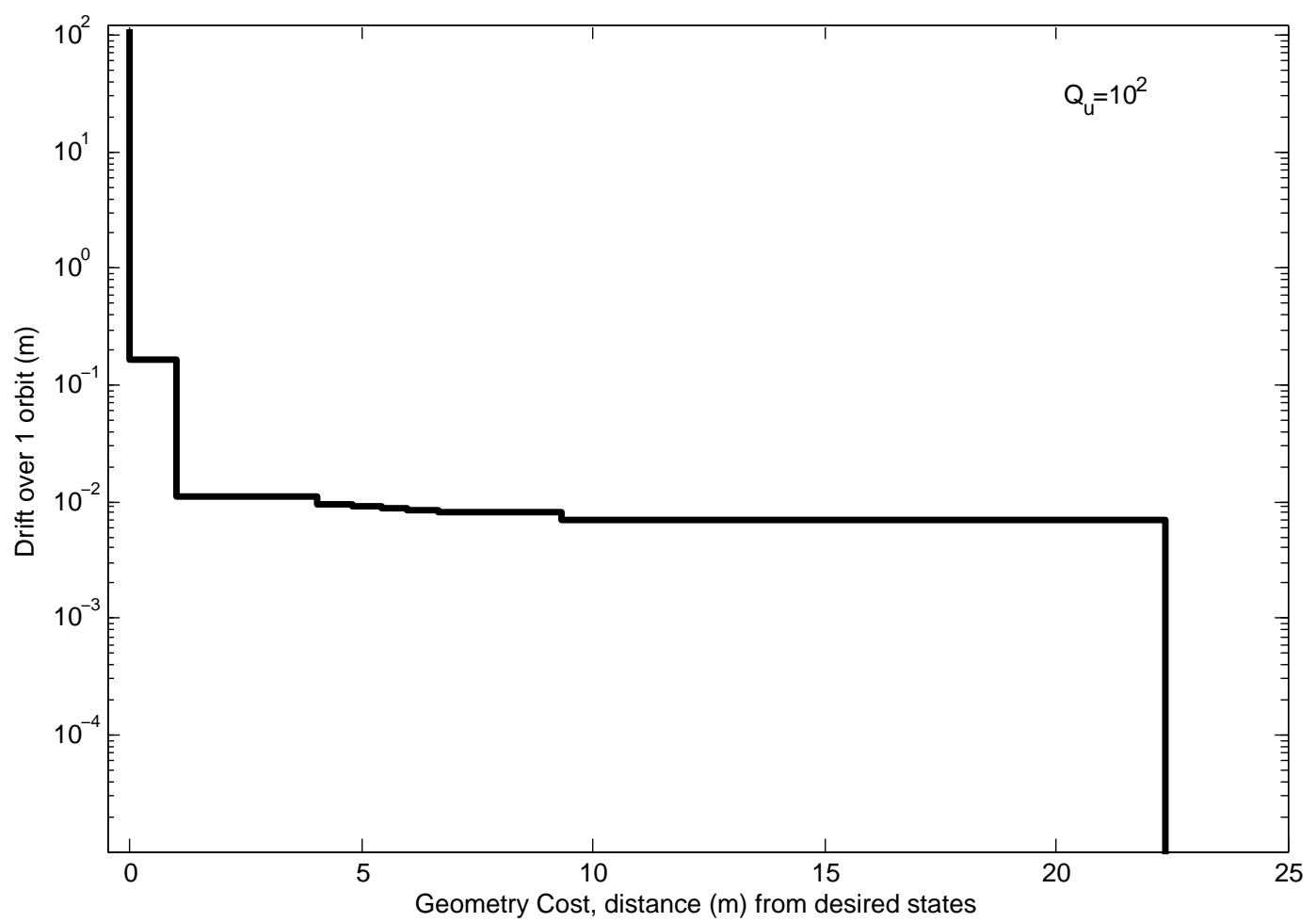

Fig. 4: Expected drift due to differential $J_{2}$ effects and geometry cost of a range of optimized initial conditions in a HEO orbit. The lines between marked points indicate the least drift that can be attained for the indicated amount of separation from the desired orbital offset.

$$
8 \text { of } 9
$$


ditions, however finds the initial conditions that require the least fuel to attain from a particular initial state. Optimized invariant initial conditions were found for a highly eccentric orbit and followed a similar pattern to those in LEO. In a formation where the principle control objective is to "not drift," the proposed approach could be used as a fuel-optimized formation flying control algorithm.

\section{Acknowledgments}

This work was funded under Cooperative Agreement NCC5-724 and Cooperative Agreement NCC5-729 through the NASA GSFC Formation Flying NASA Research Announcement. Any opinions, findings, and conclusions or recommendations expressed in this material are those of the author(s) and do not necessarily reflect the views of the National Aeronautics and Space Administration.

\section{References}

${ }^{1}$ M. Tillerson, G. Inalhan, and J.P. How, "Co-ordination and control of distributed spacecraft systems using convex optimization techniques," International Journal of Robust and Nonlinear Control, Vol.12, John Wiley \& Sons, 2002, p. 207-242.

${ }^{2}$ S.R. Vadali, K.T. Alfriend, and S. Vaddi, "Hills Equations, Mean Orbital Elements, and Formation Flying of Satellites," American Astronautical Society, AAS 00-258, March 2000.

${ }^{3}$ D.W. Gim and K.T. Alfriend, "State Transition Matrix of Relative Motion for the Perturbed Noncircular Reference Orbit," AIAA Journal of Guidance, Control, and Dynamics, vol. 26, no. 6, Nov.-Dec. 2003, p. 956-971.

${ }^{4}$ H. Schaub and K.T. Alfriend, " $J_{2}$ Invariant Relative Orbits for Spacecraft Formations," Flight Mechanics Symposium, (Goddard Space Flight Center, Greenbelt, Maryland), January 18-20, 2002, Paper No. 11.

${ }^{5}$ L.S. Breger and J.P. How, " $J_{2}$-Modified GVE-Based MPC for Formation Flying Space," AIAA GNC Conf., August 2005.

${ }^{6}$ D. Scharf, F. Hadaegh, and S. Ploen, "A Survey of Spacecraft Formation Flying Guidance and Control (Part II): Control," IEEE American Control Conference, June 2004, 2976-2985.

${ }^{7}$ D. Vallado, Fundamentals of Astrodynamics and Applications, McGraw-Hill, 1997.

${ }^{8}$ X. Duan and P.M. Bainum, "Design of Spacecraft Formation Flying Orbits," American Astronautical Society, AAS 03-588, August 2003.

${ }^{9}$ Schaub, Hanspeter and Junkins, John L., Analytical Mechanics of Space Systems, AIAA Education Series, Reston, VA, 2003.

${ }^{10}$ S. Curtis, "The Magnetospheric Multiscale Mission Resolving Fundamental Processes in Space Plasmas," NASA GSFC, Greenbelt, MD, Dec. 1999. NASA/TM2000-209883.

${ }^{11}$ D. Bertsimas and J.N. Tsitsiklas, Introduction to Linear Optimization, Athena Scientific, Belmont, 1997. 